\title{
A promising treatment for hemophilia
}

By using a technique called genome editing, scientists have successfully treated the genetic blood clotting disorder, hemophilia, in mice. These results raise the possibility of using gene therapy techniques to treat a wider range of genetic diseases in humans.

In this study, Katherine High of the Children's Hospital Philadelphia, PA, and colleagues used mice that were genetically engineered to carry a faulty version of the human gene $F 9$, which is found in people with hemophilia (Nature doi:10.1038/ nature 10177; published online 26 June 2011). F9 helps control the level of blood coagulation factor IX. The circulating levels of factor IX in most individuals with hemophilia are less than $1 \%$ of the levels of factor IX in unaffected individuals. The genetically engineered mice in this study had no detectable factor IX. High and her colleagues worked with a team at Sangamo BioSciences in Richmond, CA, to develop enzymes called zinc-finger nucleases that would target and remove

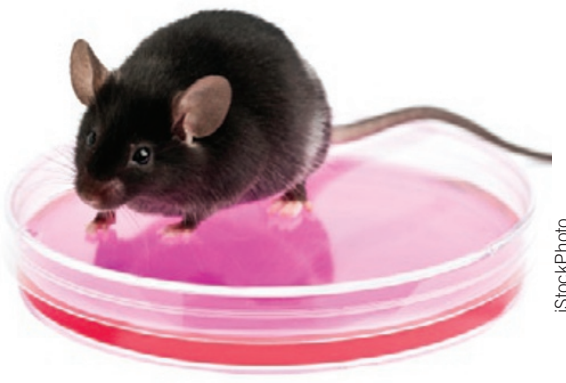

the portion of the $F 9$ gene containing $95 \%$ of $F 9$ mutations.

The research team injected an engineered viral vector carrying the zincfinger nucleases and another viral vector containing the wild-type $F 9$ sequence into mice. Both of these vectors were designed to travel to the liver. After treatment, the circulating levels of factor IX ranged from $3-7 \%$ of normal levels. Additionally, the average clotting time for mice that received the treatment was 44 seconds, which did not differ significantly from the average clotting time (36 seconds) for wild-type mice. The mice with hemophilia had average clotting times of 67 seconds.

When the researchers partially removed the livers of mice that had received the treatment, factor IX was still produced at similar levels, showing that the genome edits persisted with the division of cells. Administration of the treatment to both wild-type and hemophiliac mice appeared to have no effects on weight gain or growth in these mice. Additionally, follow-up tests at 4,29 and 32 weeks after injection showed that there were no changes in the liver function of treated mice.

Previous studies have shown that zinc-finger nucleases can be used to effectively target specific genes in cells that have been isolated and removed from the body. This study, however, represents the first time that zinc-finger nucleases have been shown to work in vivo. These results open up the possibility of using zinc-finger nucleases to treat hemophilia and other genetic diseases that affect cells that cannot be easily removed from the body.

Kirsten Dorans

\section{INDUCING SLEEP IN FRUIT FLIES}

Simply turning up the thermostat can induce sleep in genetically engineered fruit flies, researchers recently reported. By studying these flies, scientists will be able to assess the positive effects of sleep, instead of the negative effects of sleep deprivation.

Paul Shaw and colleagues from Washington University in St. Louis, MO, genetically engineered fruit flies to express a temperaturesensitive sodium channel in a brain region called the dorsal fan-shaped body (Science 232, 1571-1576; 2011). When this channel is open, the dorsal fan-shaped body becomes more excitable, signaling the rest of the brain to go to sleep. The research team found they were able to induce sleep in the genetically modified flies by raising the temperature to $31^{\circ} \mathrm{C}$ (fruit fly rooms are typically kept at $25^{\circ} \mathrm{C}$ ). They were also able to wake the sleeping flies by using a device that perturbs the flies and forces them to walk.

The team tested two competing theories of the role of sleep in memory. The first theory, synaptic homeostasis, proposes that sleep provides a homeostatic reduction in synaptic strength, reducing the intensity of neural connections that are created during the day. According to this theory, if a fly was sleep deprived, the neuronal circuits would saturate or exceed available space, thereby interfering with the fly's ability to learn. The second theory, memory consolidation, hypothesizes that during sleep, memories from the previous day are replayed and consolidated.

To test the synaptic homeostasis theory, the researchers placed flies in a social environment, where they were surrounded by many other flies. The flies were then placed in individual tubes and some of them were induced to sleep for 4 hours. The flies were then taught a courtship ritual. Two days later, only the flies that had slept before learning the courtship ritual could remember the ritual. These results support the synaptic homeostasis theory, because inducing sleep immediately after exposure to social enrichment helped restore long-term memory.

The team tested the memory consolidation theory by inducing sleep in flies for 4 hours immediately after training the flies using a protocol that does not result in long-term memory formation. Flies induced to sleep immediately after the training protocol retained a memory of this protocol for days, instead of just hours, supporting the long-term memory model. The group expects that being able to experimentally induce and control sleep should help uncover the function of sleep.

Kirsten Dorans 